\title{
The Nitric Oxide-Guanylyl Cyclase Signaling Pathway Modulates Membrane Activity States and Electrophysiological Properties of Striatal Medium Spiny Neurons Recorded In Vivo
}

\author{
Anthony R. West ${ }^{1}$ and Anthony A. Grace ${ }^{2}$ \\ ${ }^{1}$ Department of Neuroscience, Finch University of Health Sciences/The Chicago Medical School, North Chicago, Illinois 60064, and ${ }^{2}$ Departments of \\ Neuroscience and Psychiatry, Center for Neuroscience, University of Pittsburgh, Pittsburgh, Pennsylvania 15260
}

\begin{abstract}
Nitric oxide (NO)-releasing interneurons are believed to regulate the activity of striatal medium spiny neurons (MSNs) that contain the NO effector enzyme guanylyl cyclase (GC). The involvement of NO-GC signaling in modulating steady-state membrane activity of striatal MSNs was examined using in vivo intracellular recordings in rats. Intrastriatal infusion of a neuronal NO synthase inhibitor or a NO scavenger via reverse microdialysis consistently decreased the amplitude of spontaneously occurring depolarized plateau potentials (up events). Intrastriatal infusion of a NO scavenger also decreased the amplitude of EPSPs evoked during electrical stimulation of the orbital prefrontal cortex. The effect of the NO scavenger on spontaneous up events was partially reversed by coperfusion with a cell-permeable cGMP analog. Intracellular injection of MSNs with a soluble GC inhibitor resulted in large decreases in the following: (1) spontaneous up-event amplitude, (2) responsiveness to depolarizing current, (3) action potential amplitude, and (4) input resistance. These effects were partially reversed by coinjection of cGMP. Conversely, intracellular injection of a phosphodiesterase inhibitor increased MSN neuron membrane excitability. These results indicate that, in the intact animal, the NO signaling pathway exerts a powerful tonic modulatory influence over the membrane activity of striatal MSNs via the activation of GC and stimulation of cGMP production.
\end{abstract}

Key words: nitric oxide; cGMP; guanylyl cyclase; striatum; medium spiny neuron; reverse microdialysis

\section{Introduction}

Evidence has accumulated demonstrating that striatal nitric oxide (NO)-generating interneurons exert a powerful influence over striatal function (Kawaguchi, 1997; Calabresi et al., 2000a; West et al., 2002a). NO is a gaseous neurotransmitter produced by nitric oxide synthase (NOS) after NMDA receptor activation and intracellular calcium influx (Marin et al., 1992; East et al., 1996). NO diffuses freely through biological membranes and stimulates guanylyl cyclase (GC) and other effector enzymes located in presynaptic and postsynaptic elements (Garthwaite and Boulton, 1995). Within the striatal complex, type 1 (neuronal) NOS activity is primarily responsible for the generation of NO and is localized exclusively to a subclass of aspiny interneurons that colocalize somatostatin, neuropeptide Y, and GABA (Kubota et al., 1993; Kharazia et al., 1994).

Received 0ct. 1, 2003; revised Dec. 10, 2003; accepted Jan. 6, 2004.

This work was supported by United States Public Health Service MH 45156, 57440 (A.A.G.), and NS 10725; Tourette Syndrome Association; and National Alliance for Research on Schizophrenia and Depression (A.R.W.). We thank Nicole MacMurdo and Christy Wyant for their excellent technical assistance and Brian Lowry for the development of software (Neuroscope8) used in data acquisition and analysis. We also thank Drs. Stan B. Floresco and Ali Charara for their valuable assistance and comments regarding this manuscript.

Correspondence should be addressed to Dr. Anthony A. Grace, Department of Neuroscience, 446 Crawford Hall, University of Pittsburgh, Pittsburgh, PA 15260. E-mail: grace@brain.bns.pitt.edu. DOI:10.1523/JNEUROSCI.4470-03.2004

Copyright $\odot 2004$ Society for Neuroscience $\quad$ 0270-6474/04/241924-12\$15.00/0
Ultrastructural studies of striatal NOS-containing interneurons have reported that NOS-containing terminals synapse on dual-input dendritic spines of striatal medium spiny neurons (MSNs) known to contain high levels of GC, cGMP-dependent protein kinase (PKG), and other components of the cGMP signaling system (Ariano, 1983; Sancesario et al., 2000; Hidaka and Totterdell, 2001). Additionally, the close proximity of these nitrergic inputs to the dopaminergic and glutamatergic synapses of MSNs (Hidaka and Totterdell, 2001) suggests that NO transmission may play an important role in modulating the responsiveness of MSNs to afferent drive (for review, see West et al., 2002a). In support of this, recent electrophysiological studies have demonstrated that NO-GC signaling modulates corticostriatal synaptic plasticity in vitro (Calabresi et al., 1999a,b, 2000b) and glutamate-induced excitations in vivo (Di Giovanni et al., 2003). NO generation, produced after the activation of corticostriatal pathways, is also critically involved in mediating electrotonic coupling between MSNs (O'Donnell and Grace, 1997). Recent studies showing that striatal NO regulates the responsiveness of midbrain dopamine (DA) neurons to electrical stimulation of the striatum and prefrontal cortex (PFC) also indicate that NO signaling plays an important role in the integration of information transmitted to basal ganglia output centers via corticostriatal and striatal efferent pathways (West and Grace, 2000).

Currently, however, it is not known how activation of striatal 
NO-GC signaling pathways affects the steady-state membrane activity of MSNs recorded in vivo. When recorded in the intact animal, MSNs often exhibit characteristic shifts in membrane potential consisting of a depolarized plateau potential or up state and a hyperpolarized resting membrane potential termed the down state (Wilson, 1993; O'Donnell and Grace, 1995; Wilson and Kawaguchi, 1996). Although the up state is known to be driven by GLUergic afferents (Wilson, 1993; O'Donnell and Grace, 1995) and potently modulated by DA receptor activation (West and Grace, 2002a), the impact of intrinsic neuromodulatory circuits on up- and down-state membrane properties is unknown. In the current studies, we use in vivo intracellular electrophysiological recording methods and reverse microdialysis to study the role of nitrergic systems in regulating the membrane activity of striatal MSNs. The current data demonstrate that striatal NO production modulates the membrane properties of striatal MSNs in the up and down states via the activation of the GC pathway.

A portion of these results has been presented in preliminary form (West and Grace, 2001, 2002b; West et al., 2002a).

\section{Materials and Methods}

Materials. Carboxy PT-10 potassium (CPT-10), 8-bromo-cGMP (8-BrcGMP), and cGMP were purchased from Sigma (St. Louis, MO). 7-Nitroindazole sodium (7-NI) was purchased from Calbiochem (La Jolla, CA). $1 H$-[1,2,4] Oxadiazolo[4,3-a]quinoxalin-1-one (ODQ) and zaprinast were purchased from Tocris Cookson (Ellisville, MO). All of the other reagents were of the highest grade commercially available.

Subjects and surgery. Intracellular recordings of striatal neurons were obtained in vivo from male Sprague Dawley rats (Hilltop, Scottdale, PA) weighing 275-450 gm. Before experimentation, animals were housed two per cage under conditions of constant temperature $\left(21-23^{\circ} \mathrm{C}\right)$ and maintained on a $12 \mathrm{hr}$ light/dark cycle with food and water available ad libitum. All of the animal procedures were approved by the University of Pittsburgh Institutional Animal Care and Use Committee and adhere to the Guide for the Care and Use of Laboratory Animals published by the United States Public Health Service. Before surgery, animals were deeply anesthetized with chloral hydrate $(400 \mathrm{mg} / \mathrm{kg}$, i.p.) and placed in a stereotaxic apparatus (Narishige, Tokyo, Japan). The level of anesthesia was periodically verified (every $10-15 \mathrm{~min}$ ) via testing for the hindlimb compression reflex and maintained using supplemental administration of chloral hydrate $(80 \mathrm{mg} / \mathrm{ml})$ via a lateral tail vein catheter $(\sim 0.2 \mathrm{ml} / 0.5$ $\mathrm{hr})$. Temperature was monitored using a rectal probe and maintained at $37^{\circ} \mathrm{C}$ with a heating pad.

Intracellular recordings. Intracellular recording was performed using microelectrodes filled with a potassium acetate $(3 \mathrm{M})$ solution containing $2 \%$ biocytin as described previously (West et al., 2002b; West and Grace, 2002). Cell penetrations were defined as stable when the cell exhibited a resting membrane potential of at least $-60 \mathrm{mV}$, fired action potentials having amplitudes of at least $45 \mathrm{mV}$ with a positive overshoot, and fired a train of spikes in response to membrane depolarization. After impaling a neuron, membrane activity was allowed to stabilize for several minutes until a steady state was reached. Baseline membrane activity was then recorded for at least $5 \mathrm{~min}$ after which the response to intracellular injection of hyperpolarizing and depolarizing currents was determined. In some experiments, drug was injected into the cell via the recording electrode using brief depolarizing pulses ( $\sim 20-50 \mathrm{pA} ; 150 \mathrm{msec})$ delivered during cell stabilization and before the $5 \mathrm{~min}$ baseline recording period. When possible, after experimental manipulations, cells were injected with biocytin $(\sim 10-60 \mathrm{~min})$ via application of depolarizing pulses $(\sim 0.5 \mathrm{nA} ; 300 \mathrm{msec})$ through the recording electrode. After withdrawing the electrode from the cell, the extracellular electrode tip potential was recorded, and membrane potential measurements were corrected accordingly.

Data analysis. Data were analyzed off-line using Neuroscope software applications developed in our laboratory and using an Intel-based microcomputer with a data acquisition board interface (Microstar Labora-
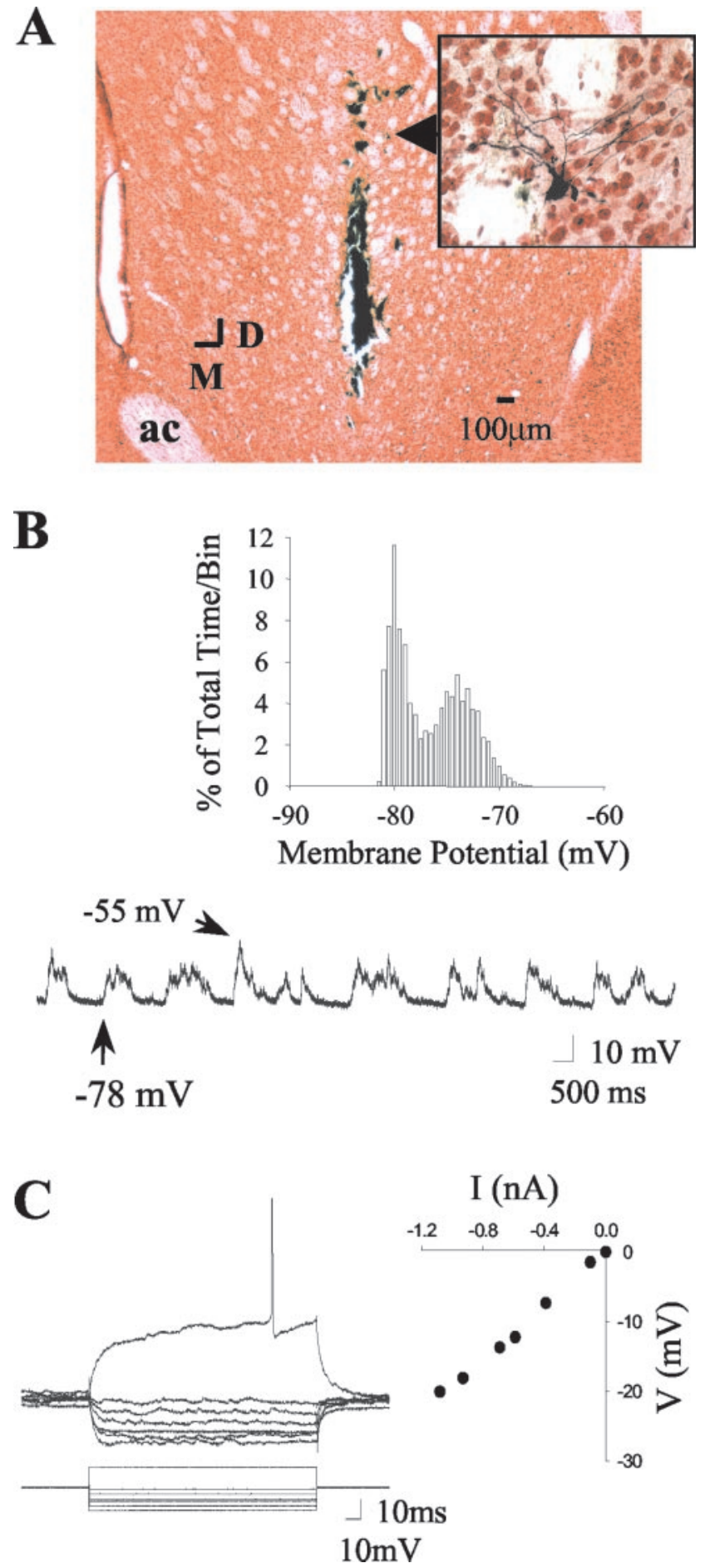

Figure 1. Intracellular recordings from striatal neurons located proximal to the microdialysis probe. $A$, Coronal section $(5 \times)$ of the striatum demonstrating the location of a striatal neuron (arrow) labeled after intracellular biocytin injection (enlarged to $40 \times$ in the inset). Note that the neuron was located proximal $(50-100 \mu \mathrm{m})$ to the active zone of the microdialysis probe (extends dorsally $4 \mathrm{~mm}$ from the termination point of the probe track). ac, Anterior commissure; $D$, dorsal; $M$, medial. $B$, Intracellular recordings from the striatal neuron labeled in $A$ revealed that this cell did not fire spontaneously but did exhibit membrane activity characterized by rapid and spontaneous transitions from a hyperpolarized state to a depolarized plateau. Inset, Example of a time interval plot of membrane potential activity ( 30 sec recordings sampled at $10 \mathrm{kHz}$ ) recorded from the neuron shown in $A$. C, Left, In the same cell, intracellular injection of 150msec-duration constant hyperpolarizing current pulses (bottom traces) induced deflections in the membrane potential (top traces). Right, A plot of the steady-state voltage deflections against the current pulse amplitudes taken from recordings shown on the left, revealing an input resistance of $18.3 \mathrm{M} \Omega$ for this neuron. 

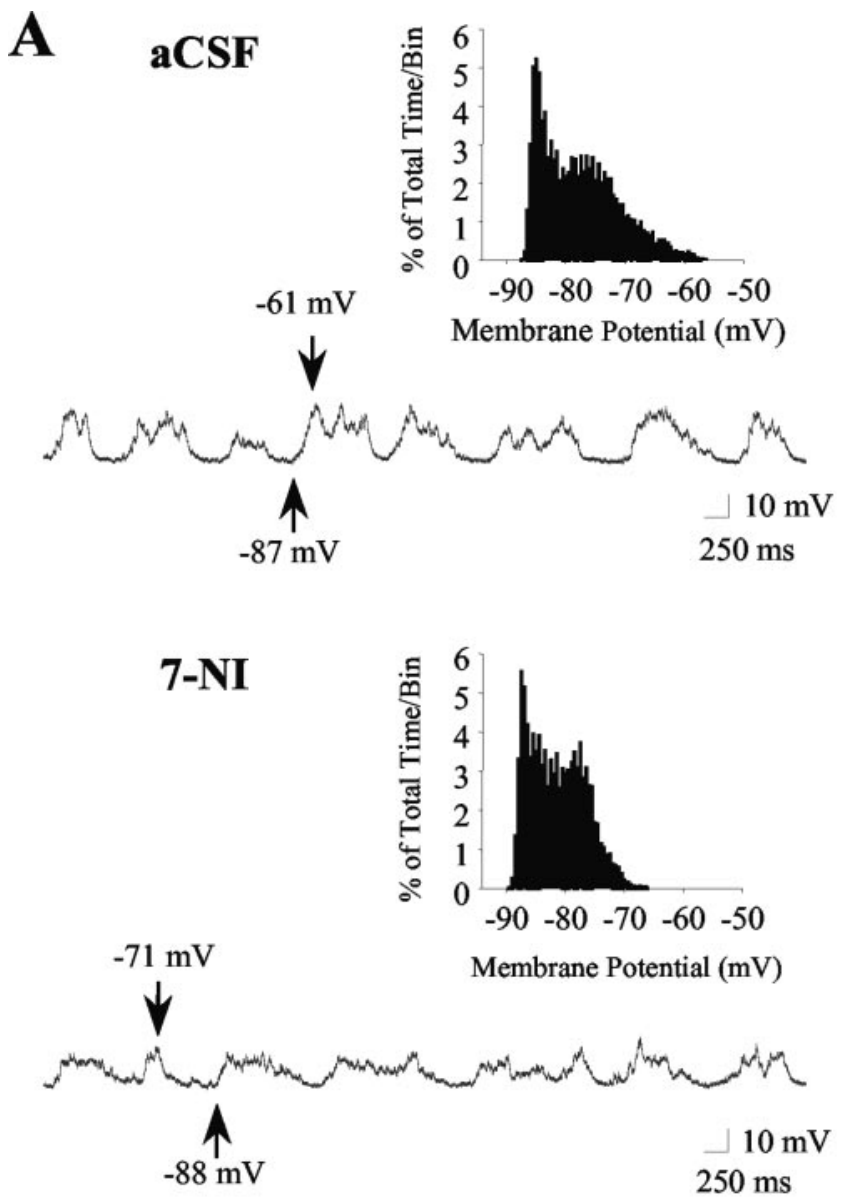

B
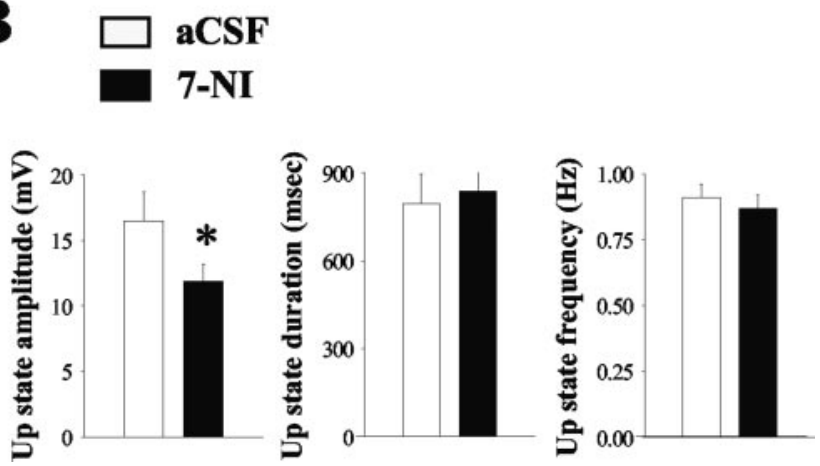

Figure 2. Intrastriatal infusion of the neuronal NOS inhibitor 7-NI decreases the amplitude of spontaneous up events in vivo. $A$, Top, During aCSF (vehicle) infusion, the majority of striatal neurons exhibited rapid spontaneous shifts in steady-state membrane potential and bimodal membrane potential distributions (inset, 30 sec recordings sampled at $10 \mathrm{kHz}$ ) in the absence of spontaneous spike discharge (top trace). Bottom, All of the neurons $(n=4)$ recorded during local 7-NI infusion (300 $\mu \mathrm{m} ; 10-60 \mathrm{~min}$ ) still exhibited up- and down-state activity (bottom trace); however, a significant decrease in the up-state amplitude was observed. Arrows indicate the membrane potential at its maximal depolarized and hyperpolarized levels. $B$, Left, The mean \pm SEM up-state amplitude was reduced after intrastriatal 7-NI infusion ( $n=4$; ${ }^{*} p<$ 0.05 ; paired $t$ test). The mean \pm SEM up-state duration (center) and frequency of occurrence (right) was not affected by intrastriatal 7-NI infusion ( $n=4 ; p>0.05$; paired $t$ test).

tories, Bellevue, WA). In within-subjects experiments combining microdialysis, basal activity and the influence of local drug infusions were determined by comparing the membrane potential activity recorded during the last $30-60 \mathrm{sec}$ of the $5 \mathrm{~min}$ artificial CSF (aCSF) (control) infusion period, with similar recordings made during drug infusions (see below). In between-subjects experiments in which drug was delivered
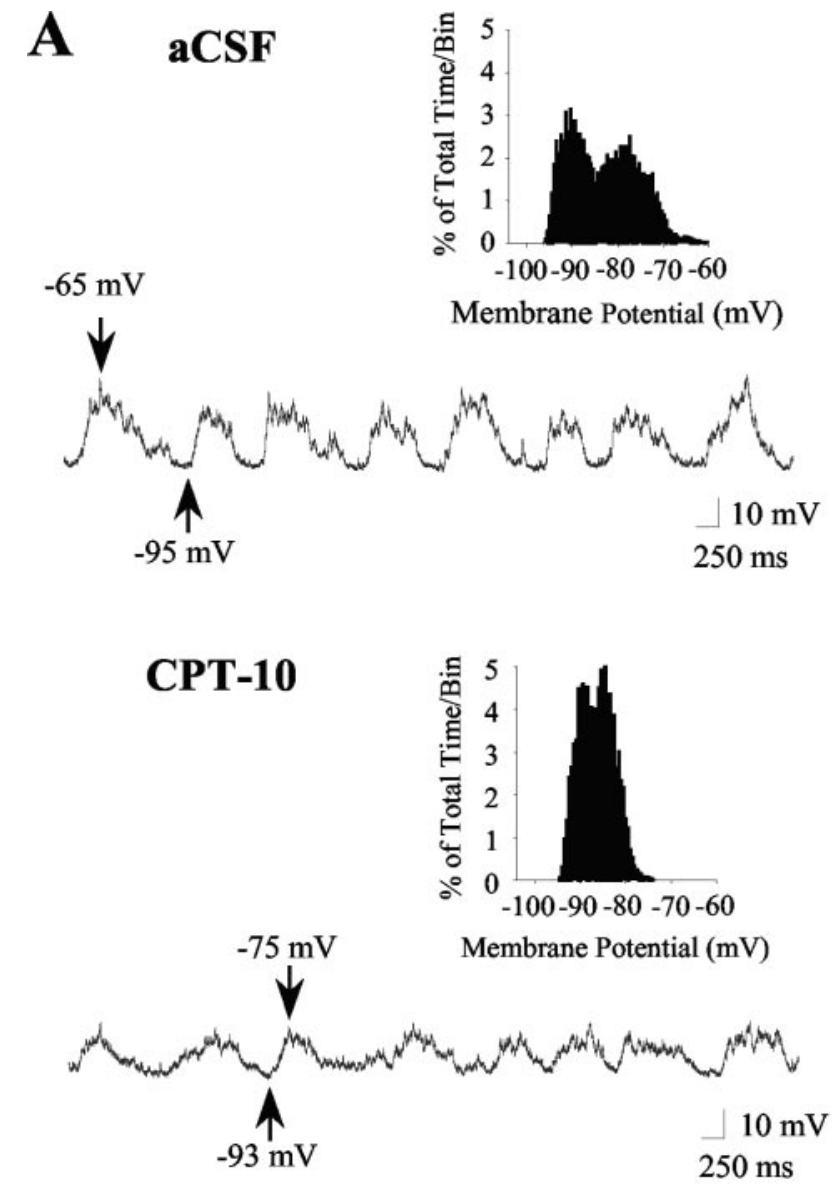

B
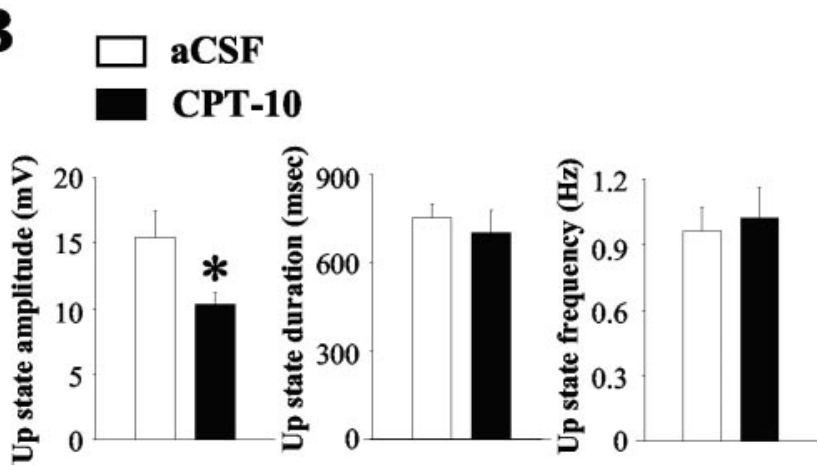

Figure 3. Intrastriatal infusion of the NO scavenger (PT-10 decreases the amplitude of up events. $A$, Top, During aCSF (vehicle) infusion, the majority of striatal neurons exhibited rapid spontaneous shifts in steady-state membrane potential and bimodal membrane potential distributions (inset, $30 \mathrm{sec}$ recordings sampled at $10 \mathrm{kHz}$ ) in the absence of spontaneous spike discharge (top trace). Bottom, All of the neurons $(n=5)$ recorded during local CPT-10 infusion (1 $\mathrm{mm} ; 5-35 \mathrm{~min}$ ) still exhibited up- and down-state activity (bottom trace); however, a significant decrease in the up-state amplitude was observed. Arrows indicate the membrane potential at its maximal depolarized and hyperpolarized levels. $B$, Left, The mean \pm SEM up-state amplitude was reduced after intrastriatal (PT-10 infusion ( $n=5$; ${ }^{*} p<0.05$; paired $t$ test). Right, The mean \pm SEM up-state duration (center) and frequency of occurrence (right) was not affected by intrastriatal (PT-10 infusion ( $n=5 ; p>0.05$; paired $t$ test).

into the striatum via reverse microdialysis or injected directly into the cell, the influence of drug was determined by comparing basal and evoked activity in experimental groups to time-matched controls injected with vehicle [0.5\% dimethylsulfoxide (DMSO)]. In all of the cases, the existence of bistable membrane activity was determined as described previously (O'Donnell and Grace, 1995; West et al., 2002b; West and Grace, 2002). Briefly, the presence of a bistable event was defined as a 


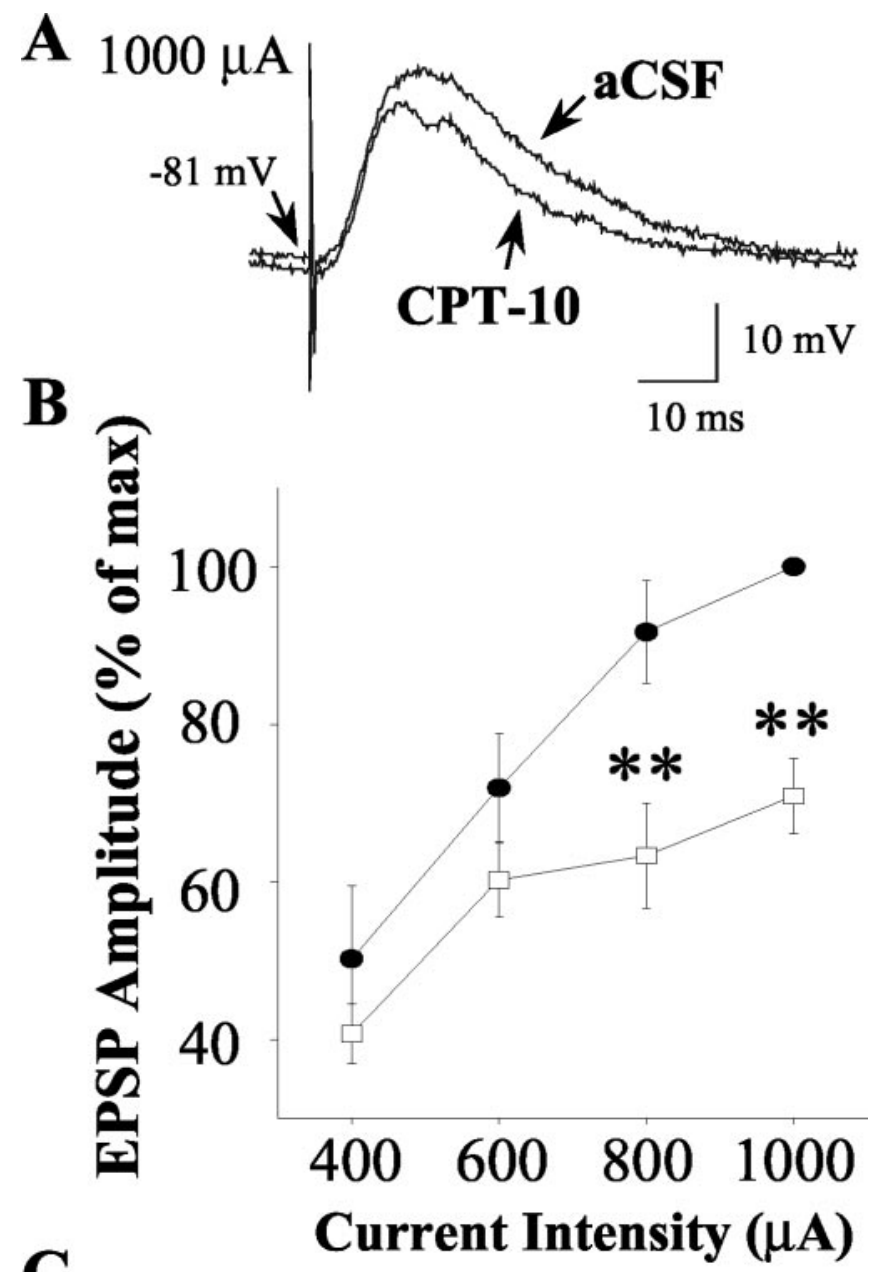

C

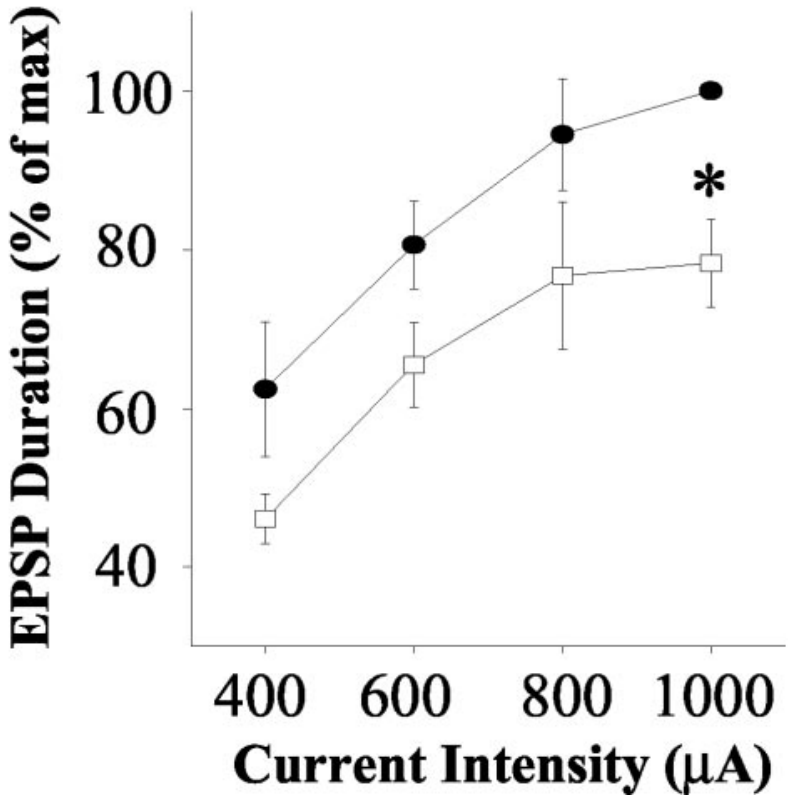

Figure 4. Intrastriatal CPT-10 infusion decreases the responsiveness of striatal neurons to electrical stimulation of the orbital prefrontal cortex. A series of single pulses $(0.2 \mathrm{~Hz})$ of electrical stimuli were delivered to the $\mathrm{OPFC}$ as described in Materials and Methods during intrastriatal aCSF or CPT-10 infusion (1 mM). A, Representative traces of maximal EPSPs evoked in the same striatal neuron during aCSF and CPT-10 infusion. B. The mean amplitude of EPSPs (expressed as percentage of maximal response) evoked by 800 and $1000 \mu \mathrm{A}$ stimulus intensities was reduced after intrastriatal CPT-10 infusion $\left(n=5 ;{ }^{* *} p<0.01\right.$; two-way repeated-measures ANOVA with Dunnett's post hoc test). C, The mean duration of EPSPs (expressed as percentage of rapid transition in membrane potential to a depolarized plateau potential exhibiting an amplitude $\geq 7 \mathrm{mV}$ (range, $8.3-32.8 \mathrm{mV}$ ), which was maintained for at least $100 \mathrm{msec}$. Time interval plots of membrane potential activity (30-60 sec recordings sampled at $10 \mathrm{kHz}$ ) recorded from neurons exhibiting bistable activity were generated. From these plots, the up- and down-state modes, average membrane potential, and maximal depolarized and hyperpolarized membrane potentials within the distribution were determined. The frequency of up (depolarized) events per $30 \mathrm{sec}$ sample was determined, with the amplitude of up events measured from the beginning of the rising phase to the peak of the depolarization plateau. The duration of up events was measured from the beginning of the rising phase to the point at which the falling phase returned to the initial baseline membrane potential. Input resistance of each neuron in the down state was calculated by injecting a series of hyperpolarizing current pulses intracellularly (150 msec; 0.1-1.5 $\mathrm{nA}$ ) and plotting the resulting membrane deflections against the amplitude of the current pulse (see Fig. $1 C$, right). The resulting data points were then fitted to a least squares regression line, and the input resistance was estimated from the slope of the lines. Rheobase current was defined as the minimum depolarizing current required to evoke a spike. The spike threshold, spike amplitude, spike duration, spike overshoot, and afterhyperpolarization were determined from the first spike evoked at each depolarizing current level. The spike threshold was visually identified as the change in slope evident at the transition from the graded depolarization to the onset of the rapid depolarizing phase of the spike. Spike amplitude was defined as the difference in voltage between the spike threshold and spike peak. Spike duration was measured from the spike threshold to the point at which the falling phase of the action potential returned to the membrane potential at spike threshold. The afterhyperpolarization was measured from the spike threshold membrane potential to the peak hyperpolarization reached during the falling phase of the action potential. EPSP amplitudes were measured from the beginning of the rising phase to the apex of the depolarization. EPSP duration was measured from the beginning of the rising phase to the point at which the falling phase returned to the initial pre-rising-phase membrane potential. In studies using single-pulse stimulation protocols, data were expressed as percentage of maximal response evoked by $1000 \mu \mathrm{A}$ stimulus intensities. In paired-pulse studies, data were expressed as percentage of the amplitude of the first (control) EPSP of the pair evoked by $1000 \mu \mathrm{A}$ stimulus intensities. The statistical significance of drug-induced changes in measures of cell activity before and after drug administration was determined using a paired $t$ test or two-way repeated measures ANOVA in withinsubjects studies, or a $t$ test or ANOVA with post hoc Dunnett's or Dunn's test in between-subjects studies as indicated.

Electrical stimulation. In each experiment, twisted-pair bipolar stimulating electrodes (Plastics One, Roanoke, VA) were implanted into the orbital prefrontal cortex (oPFC) (coordinates, 3.5-4.2 $\mathrm{mm}$ anterior to bregma; $0.8-2.2 \mathrm{~mm}$ lateral to midline; $3.0-4.5 \mathrm{~mm}$ ventral to brain surface) ipsilateral to the recording electrode. Stimulation sites in the medial, ventral, and ventrolateral orbital PFC were selected on the basis of the results of striatal retrograde and anterograde tracing (Deniau et al., 1996) and electrophysiological (West and Grace, 2000, 2002; West et al., 2002b) studies. Single or paired pulses (interstimulus interval, 50-100 $\mathrm{msec}$ ) of electrical stimuli with durations of 200-250 $\mu$ sec and intensities between 0.2 and $1.0 \mathrm{~mA}$ were generated using a Grass stimulator (S88) and photoelectric constant current/stimulus isolation unit (PSIU6F; Grass Instruments, Quincy, MA) and delivered at a frequency of $0.2 \mathrm{~Hz}$.

Simultaneous microdialysis and intracellular recording. Concentric microdialysis probes (Bioanalytical Systems, West Lafayette, IN) having 3-4 mm of exposed membrane (diameter, $320 \mu \mathrm{m}$; permeability, $\sim 6000$ Da) were implanted into the dorsal striatum (coordinates, $0.1-0.7 \mathrm{~mm}$ anterior to bregma; $2.0-3.5 \mathrm{~mm}$ lateral to midline; $5.5-6.5 \mathrm{~mm}$ ventral to

maximal response) evoked by $1000 \mu \mathrm{A}$ stimulus intensities was reduced after intrastriatal CPT-10 infusion ( $n=5$; ${ }^{*} p<0.05$; two-way repeated-measures ANOVA with Dunnett's post hoc test). The mean onset latency of evoked EPSPs and membrane potential before stimulation were not significantly different during aCSF or CPT-10 infusion (data not shown). Filled circles indicate the mean \pm SEM EPSP amplitude-duration recorded during aCSF (control) infusion. Open squares indicate the mean \pm SEM EPSP amplitude-duration after CPT-10 infusion. 
$\mathbf{A}$
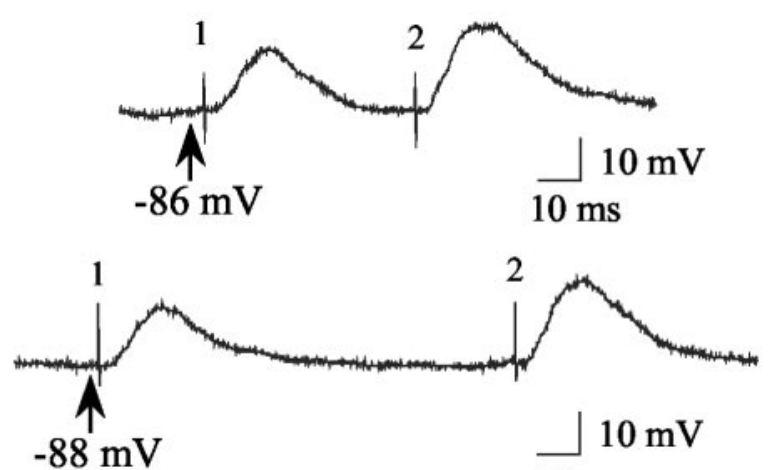

B
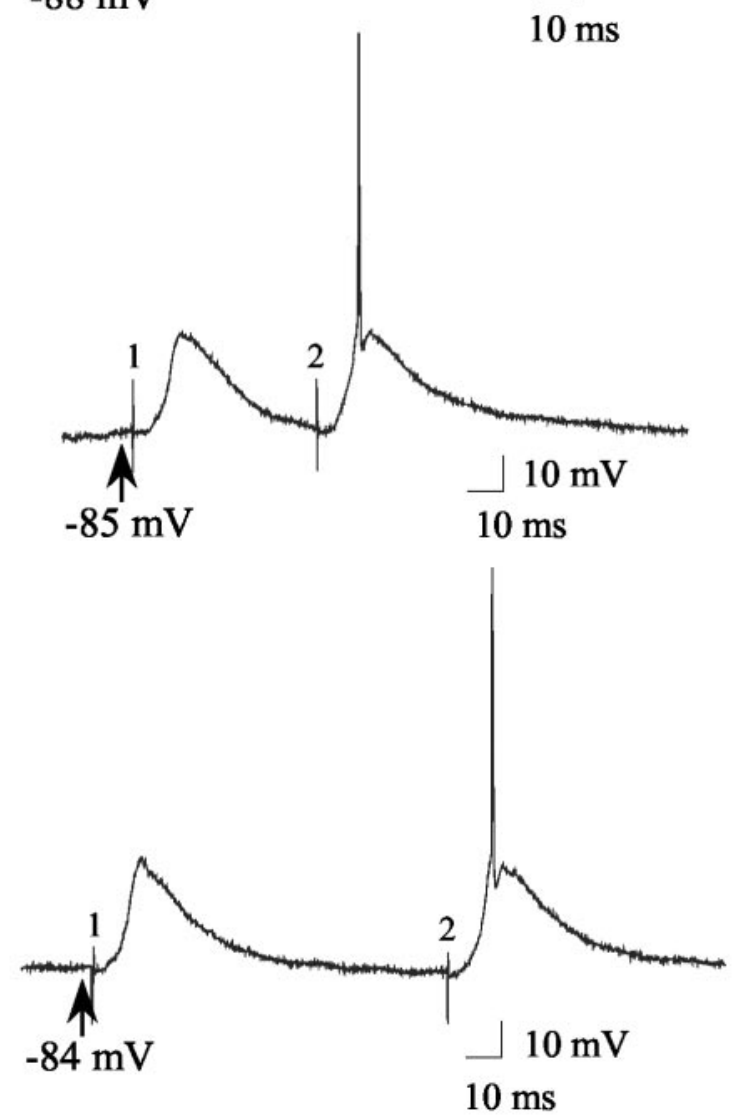

C

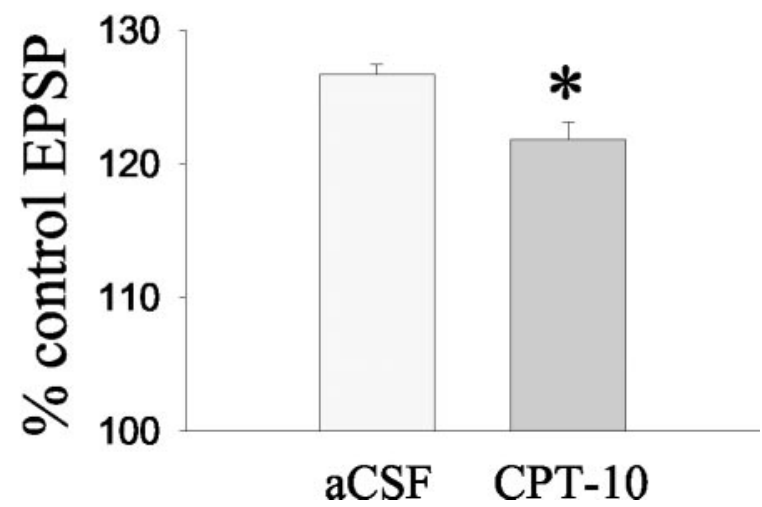

Figure 5. Intrastriatal infusion of CPT-10 decreases paired-pulse facilitation of EPSPs evoked by electrical stimulation of the orbital prefrontal cortex. A series of paired pulses $(0.2 \mathrm{~Hz}, 1 \mathrm{~mA}$ stimulus intensity; 50 or 100 msec interstimulus interval) of electrical stimuli were delivered to the $\mathrm{OPFC}$ as described in Materials and Methods during either intrastriatal aCSF or CPT-10 infusion (1 mm). A, Examples of responses evoked by paired-pulse stimulation delivered at 50 (top) and 100 (bottom) msec interstimulus intervals in the same striatal neuron during (PT-10 brain surface) over a $25-30 \mathrm{~min}$ period (3-4 $\mu \mathrm{m} / \mathrm{sec}$ ) as described previously (West et al., 2002b; West and Grace, 2002). After implantation, probes were perfused with aCSF containing (in $\mathrm{mM}$ ): $145 \mathrm{NaCl}, 2.7 \mathrm{KCl}$, $1.0 \mathrm{MgCl}_{2}, 1.2 \mathrm{CaCl}_{2}, 2.0 \mathrm{NaH}_{2} \mathrm{PO}_{4}$, and $2.0 \mathrm{Na}_{2} \mathrm{HPO}_{4}$, at a rate of 2 $\mu \mathrm{l} / \mathrm{min}$ using a Bioanalytical Systems Baby Bee microperfusion pump as described previously (West and Galloway, 1997). Electrophysiological recordings were initiated $\sim 2-4 \mathrm{hr}$ after probe implantation. Electrodes were positioned to enter the brain surface $\sim 1 \mathrm{~mm}$ lateral to the probe and lowered at a $10^{\circ}$ angle. The distance between the recording electrode at the surface of the brain and its final position near the center of the exposed length of the dialyzing membrane was estimated to be $\sim 4.6 \mathrm{~mm}$. The changeover from aCSF to drug infusion during the microdialysis procedure was accomplished using a liquid switch (Carnegie Medicine/ Bioanalytical Systems). Once drug was on board for $\sim 5 \mathrm{~min}$, basal membrane activity before and during intracellular current injection was recorded in the presence of drug. It is estimated that the time elapsed between the switch from aCSF to drug and the beginning of drug infusion into the brain was $\sim 4 \mathrm{~min}$ (taking into account the dead space in the microdialysis inlet tubing). To ensure that drug was being delivered into the brain during a given recording period, the dialysis tubing dead space $(8 \mu \mathrm{l})$ and perfusate flow rate $(2 \mu \mathrm{l} / \mathrm{min})$ were taken into account, and syringes containing drug were switched 4 min before the initiation of basal activity assessment. All of the drugs delivered via reverse microdialysis were soluble in aCSF. Effective doses of CPT-10, 7-NI, 8-Br-cGMP, cGMP, zaprinast, and ODQ were derived from previous studies (West and Galloway, 1997; Wexler et al., 1998; Calabresi et al., 1999a,b, 2000b; West and Grace, 2000; West et al., 2002a).

Histology. After experimentation, animals were deeply anesthetized and perfused transcardially with ice-cold saline followed by $4 \%$ paraformaldehyde in $0.1 \mathrm{M}$ PBS. Brains were then removed and postfixed in $4 \%$ paraformaldehyde-PBS for at least 1 week. After this period, brains were immersed in PBS-sucrose solution (25\%) until saturated. The tissue was sectioned into $60 \mu \mathrm{m}$ coronal slices, mounted, and stained with cresyl violet to enable histological determination of stimulating- and recording-electrode sites. In cases in which cells were injected intracellularly with biocytin through the recording electrode, tissue sections were processed for biocytin immunoreactivity as described previously (West et al., 2002b).

\section{Results}

Intracellular recordings were made from 59 striatal neurons recorded in 40 rats. From the above groups, 23 biocytin-stained neurons (39\%) were recovered and localized to the dorsal striatum. In experiments combining intracellular recordings and microdialysis, the somata of all of the recovered neurons were estimated to lie within a distance of $\sim 500 \mu \mathrm{m}$ from the microdialysis probe track (Fig. 1). In several cases, biocytin-immunoreactive processes were found to lie in close proximity to the microdialysis probe track $(<50 \mu \mathrm{m})$.

\section{Electrode and microdialysis probe placement}

In cells responding to synaptic activation, all of the stimulatingelectrode tips implanted into the cortex were confirmed to lie in the PFC between $\sim 3.5$ and $4.2 \mathrm{~mm}$ anterior to bregma, 0.8 and $2.2 \mathrm{~mm}$ lateral to the midline, and 3.0 and $4.5 \mathrm{~mm}$ ventral to the dural surface (Paxinos and Watson, 1986). In experiments com-

$\leftarrow$

infusion. $B$, Examples of paired-pulse facilitation of spike activity evoked during electrical stimulation [delivered at 50 (top) and 100 (bottom) msec interstimulus intervals] of corticostriatal pathways during CPT-10 infusion. The delivery of the first and second pulses of the pair is indicated by the numbers 1 and 2 , respectively. Solid arrows indicate the membrane potential before stimulation. C, The mean \pm SEM amplitude of EPSPs [expressed as percentage of the amplitude of the first (control) EPSP of the pair] evoked by $1000 \mu$ A stimulus intensities was reduced after intrastriatal CPT-10 infusion compared with control cells recorded during aCSF infusion ( $n=3-4 ;{ }^{*} p<0.05 ; t$ test $)$. 
bining intracellular recordings and microdialysis, identified probe tips were confirmed to lie within the dorsal striatum between $\sim 0.5 \mathrm{~mm}$ posterior and $1.9 \mathrm{~mm}$ anterior to bregma, 2.0 and $4.2 \mathrm{~mm}$ lateral to the midline, and 5.0 and $7.5 \mathrm{~mm}$ ventral to the dural surface (Paxinos and Watson, 1986). In cases in which biocytin-stained neurons or recording-electrode tracks could be identified, they were observed to lie within the striatal coordinates reported for the above probe tip placements in the vicinity of the dialysis probe track $(<0.5$ $\mathrm{mm}$ ). Moreover, the observation that the majority of striatal neurons tested responded to local drug infusions also demonstrated that the soma or dendritic processes of the recorded neuron came into contact with the perfused drug. Given that the current study and others have shown that neurons recorded in the vicinity of the microdialysis probe exhibit electrophysiological characteristics similar to their counterparts in the intact animal, it is unlikely that the dialysis procedure significantly impacts on the natural activity of the recorded neuron (West et al., 2002b; West and Grace, 2002).

\section{Effects of disrupting striatal nitrergic signaling on the basal and evoked activity of MSNs recorded in vivo} To determine the effects of endogenous NO tone on spontaneous membrane activity, MSNs were recorded before and after local perfusion of the neuronal NOS inhibitor 7-NI $(300 \mu \mathrm{M})$ or the NO scavenger CPT-10 (1 mM). In these withinsubjects studies, successful recordings of activity before and after drug infusions were obtained for $n=9$ cells (nine rats). The majority of the neurons in this group (eight of nine) did not exhibit spontaneous action potential discharge during aCSF or NO antagonist infusion. Intrastriatal NO antagonist infusion did not have a significant impact on up-state event duration or frequency (Figs. 2, 3) $(p>0.05$; paired $t$ test). However, the amplitude of spontaneously occurring up events was significantly reduced during $\mathrm{NO}$ antagonist infusion (Figs. 2, 3) $(p<0.05$; paired $t$ test).

To examine the potential effects of electrical stimulation of the oPFC on cells during aCSF and CPT-10 infusion (five cells; five rats), a series of single or paired pulses of stimuli $(200 \mu \mathrm{sec} ; 0.2 \mathrm{~Hz})$ were delivered at gradually increasing stimulus intensities $(0.2-1.0 \mathrm{~mA})$. Stimuli delivered using higher current amplitudes $(0.8-1.0 \mathrm{~mA})$ evoked EPSPs exhibiting rapid-onset latencies $(\sim 3-5 \mathrm{msec})$ that typically reached maximal amplitude and did not increase additionally when higher amplitude pulses were delivered. Analyses of EPSP characteristics revealed that intrastriatal CPT-10 infu-

B

CPT-10

C
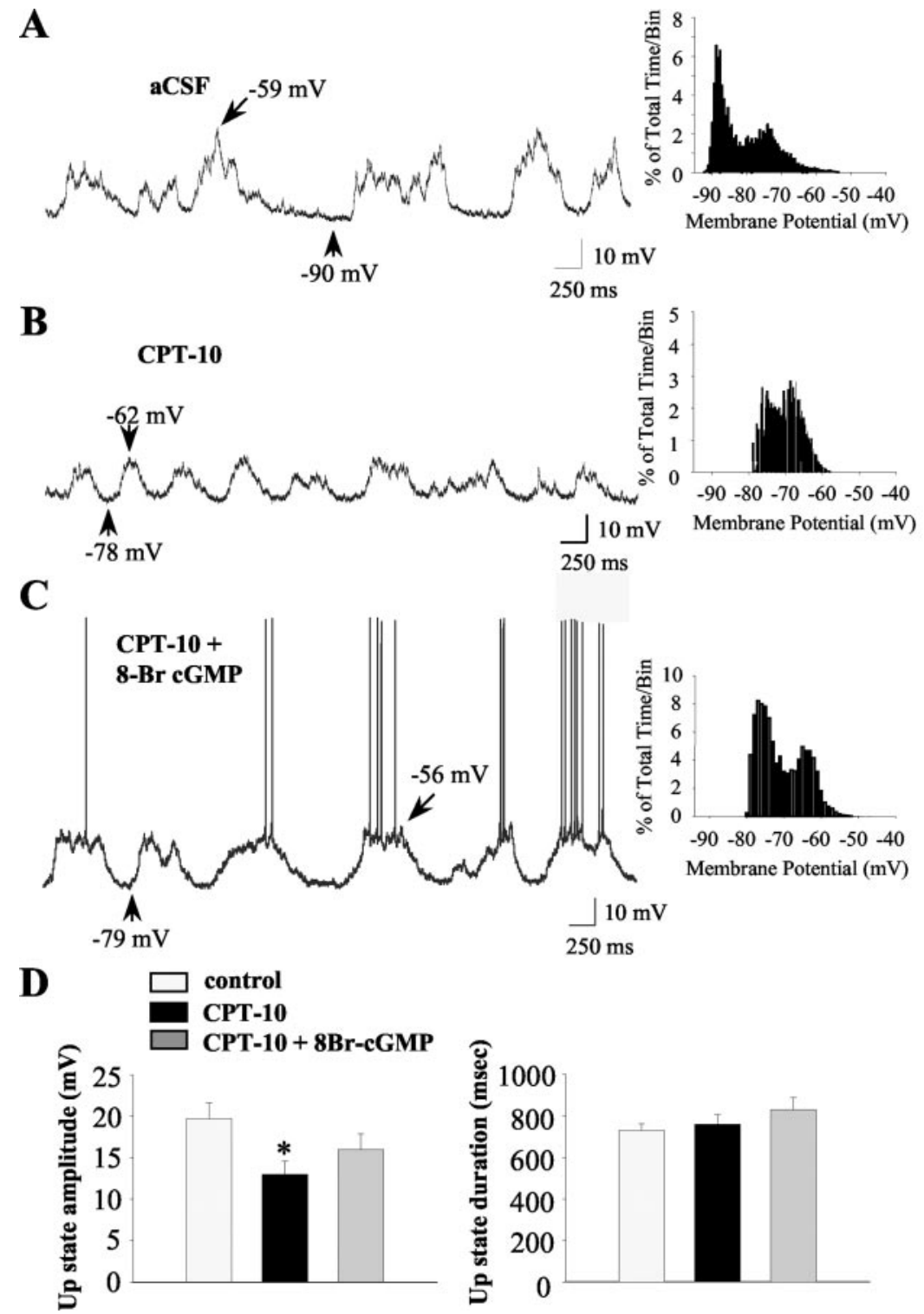

Figure 6. Intrastriatal infusion of CPT-10 modulates the membrane activity of striatal neurons in manner that is partially reversed by coperfusion with a cell-permeable cGMP analog. Striatal neurons were recorded after intrastriatal infusion of either aCSF (control), the N0 scavenger CPT-10 (1 mM), or CPT-10 (1 mM) plus the cell-permeable cGMP analog 8-Br-cGMP (2 mM). A, Left, After aCSF infusion (5 min), striatal neurons $(n=11)$ exhibited typical rapid spontaneous shifts in steady-state membrane potential. Right, Time interval plots of membrane potential activity recorded from control neurons demonstrated bimodal membrane potential distributions indicative of bistable membrane activity. $B$, Left, Similar to within-subjects studies, striatal neurons recorded after (PT-10 infusion (5 min) exhibited significantly lower amplitude up events compared with controls. Right, Time interval plots of membrane potential activity recorded from neurons recorded after CPT-10 infusion demonstrated bimodal membrane potential distributions. C, Left, Striatal neurons recorded after CPT-10 and 8-Br-cGMP infusion (5 min) exhibited high amplitude up events and higher rates of spontaneous spike discharge. Right, The membrane potential distribution of neurons recorded after CPT-10 and 8-Br-cGMP infusion was more depolarized than controls (Table 1). D, Left, The mean \pm SEM up-state amplitude was reduced after CPT-10 infusion in a manner that was partially reversed by inclusion of 8-Br-cGMP in the perfusion buffer ( $n=8-11$ cells; ${ }^{*} p<0.05$; ANOVA with Dunnett's post hoc test). Right, The mean \pm SEM up-state duration was not significantly altered by either drug treatment ( $n=8-11$ cells; $p>0.05$; ANOVA with Dunnett's post hoc test).

sion produced a decrease in the amplitude of EPSPs evoked at higher $(0.8$ and $1.0 \mathrm{~mA})$ current intensities (Fig. $4 A, B)(F=8.5$; $p<0.05$; two-way repeated-measures ANOVA with Dunnett's post hoc test; $p<0.01 ; n=5)$. Additionally, a decrease in EPSP duration evoked by $1000 \mu \mathrm{A}$ pulses was observed after intrastriatal CPT-10 infusion (Fig. $4 A, C)(F=5.9$; $p<0.05$; two-way repeated-measures ANOVA with Dunnett's post hoc test; $p<$ $0.05 ; n=5)$. There were no significant differences in the average 
Table 1. Effects of intrastriatal infusions of a NO scavenger alone and in combination with 8-Br-cGMP on the membrane properties of striatal neurons

\begin{tabular}{|c|c|c|c|}
\hline Membrane properties & Control & CPT-10 & $\begin{array}{l}\text { CPT-10 + } \\
\text { 8-Br-CGMP }\end{array}$ \\
\hline Firing rate $(\mathrm{Hz})$ & $0.05 \pm 0.03$ & $0.07 \pm 0.07$ & $0.44 \pm 0.34$ \\
\hline Average MP (mV) & $-79.3 \pm 1.6$ & $-79.1 \pm 2.0$ & $\begin{array}{c}-72.8 \pm 1.9^{*} \\
\quad(p<0.05)\end{array}$ \\
\hline Up-state frequency (Hz) & $0.96 \pm 0.04$ & $0.95 \pm 0.05$ & $0.96 \pm 0.08$ \\
\hline Maximal depolarized MP ${ }^{a}(\mathrm{mV})$ & $-54.8 \pm 3.6$ & $-64.1 \pm 3.0$ & $-53.8 \pm 5.3$ \\
\hline Up-state mode (mV) & $-75.8 \pm 2.1$ & $-77.3 \pm 2.1$ & $-69.4 \pm 2.6$ \\
\hline Down-state mode (mV) & $-84.9 \pm 1.8$ & $-82.8 \pm 1.7$ & $\begin{array}{c}-75.8 \pm 1.9^{*} \\
\quad(p<0.05)\end{array}$ \\
\hline Maximal hyperpolarized MP (mV) & $-89.7 \pm 1.5$ & $-87.4 \pm 1.9$ & $\begin{array}{c}-81.0 \pm 1.7^{*} \\
\quad(p<0.05)\end{array}$ \\
\hline Input resistance (m $\Omega$ ) & $28.3 \pm 2.1$ & $\begin{array}{l}20.3 \pm 2.5^{*} \\
(p<0.05)\end{array}$ & $32.7 \pm 5.6$ \\
\hline \multicolumn{4}{|l|}{ Action potential } \\
\hline Amplitude (mV) & $64.1 \pm 1.9$ & $67.4 \pm 2.2$ & $58.5 \pm 3.2$ \\
\hline Overshoot (mV) & $+20.7 \pm 2.9$ & $+18.6 \pm 3.2$ & $+13.4 \pm 3.6$ \\
\hline Duration (msec) & $1.1 \pm 0.04$ & $1.0 \pm 0.04$ & $1.2 \pm 0.10$ \\
\hline Threshold (mV) & $-43.4 \pm 1.7$ & $-48.6 \pm 2.1$ & $-44.8 \pm 3.3$ \\
\hline $\mathrm{AHP}(\mathrm{mV})$ & $-14.0 \pm 1.1$ & $-14.2 \pm 0.8$ & $-10.2 \pm 1.5$ \\
\hline
\end{tabular}

All data are means \pm SEM from $n=8-11$ neurons. MP, Membrane potential; AHP, afterhyperpolarization.

*Statistical significance was determined by comparing aCSF control to drug groups using an ANOVA with Dunnett's post hoc test.

${ }^{a}$ This calculation does not include membrane potential fluctuations contributed by action potentials.

onset latency of EPSPs or the membrane potential before electrical stimulation ( $p>0.05$; data not shown).

The impact of intrastriatal CPT-10 infusion on paired-pulse facilitation of synaptic responses was also examined in betweensubjects studies (seven cells; five rats). Pairs of equal-amplitude stimuli $(1.0 \mathrm{~mA})$ delivered at interpulse intervals of 50 and 100 msec produced consistent enhancements in the second of two synaptic responses in a subpopulation of neurons recorded during either aCSF or CPT-10 infusion (Fig. 5A). Neurons in both groups also occasionally exhibited paired-pulse facilitation of spike activity in response to pairs of electrical stimuli delivered at interpulse intervals of 50 and $100 \mathrm{msec}$ (Fig. 5B). Comparisons of EPSP ratios across groups revealed that neurons recorded during intrastriatal CPT-10 infusion exhibited smaller facilitatory synaptic responses (paired-pulse ratios) to paired stimuli compared with control neurons (Fig. $5 C)(p<0.05$; $t$ test; $n=3-4)$.

To control for potential recording-time effects on membrane activity, increase the drug concentration at the recording site, and allow for a more thorough examination of the effects of NO antagonism on the membrane activity of MSNs in a larger population of neurons, additional studies were performed using a between-subjects design. In these studies, basal and evoked activity were recorded intracellularly in vivo in control neurons (aCSF; $n=11$ ) and cells recorded during intrastriatal infusion of the NO scavenger CPT-10 administered alone $(n=10)$ or in combination with the cell-permeable cGMP analog 8-Br-cGMP $(n=8)$. Striatal neurons in both aCSF control and drug groups often exhibited spontaneous shifts in membrane potential from a hyperpolarized state to a depolarized plateau (Fig. 6). Intrastriatal CPT-10 infusion did not affect the frequency or duration of spontaneous up-state events (Fig. 6A,B; Table 1) ( $p>0.05)$. However, as observed in within-subjects studies, the amplitude of spontaneously occurring up events was significantly reduced in neurons recorded during CPT-10 infusion (Fig. 6A, $B$; Table 1) $(F=3.7 ; p<0.05$; ANOVA with Dunnett's post hoc test; $p<$ $0.05)$. Examination of the responsiveness of neurons to hyperpolarizing current injection delivered in the down state revealed that CPT-10 infusion also induced a significant decrease in input resistance (Table 1$)(F=4.1 ; p<0.05$; ANOVA with Dunnett's post hoc test; $p<0.05)$, which was reversed by coperfusion with 8-Br-cGMP (Table 1) ( $p>0.05$ ). Additionally, neurons recorded during coperfusion of CPT-10 and 8-Br-cGMP exhibited significantly more depolarized membrane potentials compared with neurons recorded in the drug-free state (Table 1) $(F=3.7$; $p<0.05$; ANOVA with Dunnett's post hoc test; $p<0.05)$. There was no significant effect of either drug treatment on action potential characteristics evoked by depolarizing current pulses (Table 1) $(p>0.05)$.

\section{Effects of intracellular manipulations of cGMP levels on basal} and evoked activity of striatal MSNs

To examine the influence of tonically active GC on MSN membrane activity, basal and evoked activity were recorded intracellularly in vivo in control neurons (0.5\% DMSO; $n=6)$ and neurons injected with the GC inhibitor ODQ $(100 \mu \mathrm{M} ; n=5)$ alone or in combination with cGMP $(1 \mathrm{mM} ; n=5)$. Additionally, the influence of the phosphodiesterase (PDE) inhibitor zaprinast (200 $\mu \mathrm{M} ; n=5)$ on membrane activity was assessed in a similar manner. To control for potential recording-time effects, measurements were taken at similar time points after injection of vehicle or drug.

Intracellular ODQ injection did not affect the frequency or duration of spontaneous up-state events (Fig. 7A, $B$; Table 2) $(p>0.05)$. However, both the amplitude of spontaneously occurring up events (Fig. $7 A, B)(F=5.1 ; p<0.01$; ANOVA with Dunnett's post hoc test; $p<0.05)$ and the maximal depolarized membrane potential recorded in the up state (Table 2$)(F=15.8$; $p<0.0001$; ANOVA with Dunnett's post hoc test; $p<0.05)$ were significantly reduced after ODQ injection. Examination of the responsiveness of neurons to hyperpolarizing current injection delivered in the down state revealed that ODQ treatment induced a significant decrease in input resistance (Table 2) $(Q=2.7 ; p<$ 0.05 ; ANOVA with Dunn's post hoc test; $p<0.05$ ). Furthermore, ODQ injection induced a significant increase in the average minimal amplitude of depolarizing current required to elicit action potential discharge (rheobase) compared with neurons recorded in the presence of vehicle (Fig. $8 A, B)(F=8.5 ; p<0.01$; ANOVA with Dunnett's post hoc test; $p<0.05)$. There was no significant 
effect of ODQ on membrane potential before current injection (Fig. $8 B)(p>0.05)$. A significant decrease in the amplitude of the action potential was also observed in neurons recorded during ODQ injection in the absence of other effects on spike characteristics (Table 2) $(F=3.8 ; p<0.05$; ANOVA with Dunnett's post hoc test; $p<$ 0.05). In experiments in which cGMP (1 $\mathrm{mM}$ ) was added to the recording electrolyte along with ODQ, no significant differences in membrane potential characteristics, passive membrane properties, or evoked activity were observed compared with controls (Table 2) $(p>0.05)$, indicating that the inclusion of cGMP reversed the effects of ODQ on basal and evoked activity. Additionally, neurons recorded after cGMP and ODQ injection exhibited significantly longer up-state durations compared with controls (Fig. 7C,E) $(F=$ 4.0; $p<0.05$; ANOVA with Dunnett's post hoc test; $p<0.05)$. This effect was also observed in neurons recorded after intracellular injection of the PDE inhibitor zaprinast (Fig. $7 D, E)(F=4.0 ; p<0.05$; ANOVA with Dunnett's post hoc test; $p<$ $0.05)$, indicating that augmentation of intracellular cGMP levels enhances the duration of naturally occurring up events. In contrast to ODQ, intracellular injection of the PDE inhibitor zaprinast robustly depolarized the membrane of MSNs and increased the spontaneous spike activity of these cells (Fig. 7, Table 2) $(Q=2.9$; $p<$ 0.05; ANOVA with Dunn's post hoc test; $p<0.05)$. Rheobase current could not be determined for the zaprinast group, because the firing rate of some of these neurons was $>1.0 \mathrm{~Hz}$ after injection of drug.

\section{Discussion}

The results of the current study indicate that, in the intact animal, tonic NO modulates the spontaneously occurring upand down-state membrane activity of striatal MSNs via the GC-cGMP signaling pathway. Overall, these findings are in agreement with our previous observations showing that intrastriatal infusion of a NO generator increased the spontaneous firing rate of striatal neurons recorded extracellularly in vivo (West et al., 2002a). The current study greatly extends these findings by revealing that endogenous $\mathrm{NO}-\mathrm{GC}$ signaling modulates the up- and down-state membrane properties and synaptic responses of striatal neurons exhibiting electrophysiological and morphological characteristics of MSNs. Thus, pharmacological disruption of endogenous NO-GC signaling via the local infusion of a $\mathrm{NO}$ antagonist or intracellular application of a GC inhibitor decreased the
$\mathbf{A}$
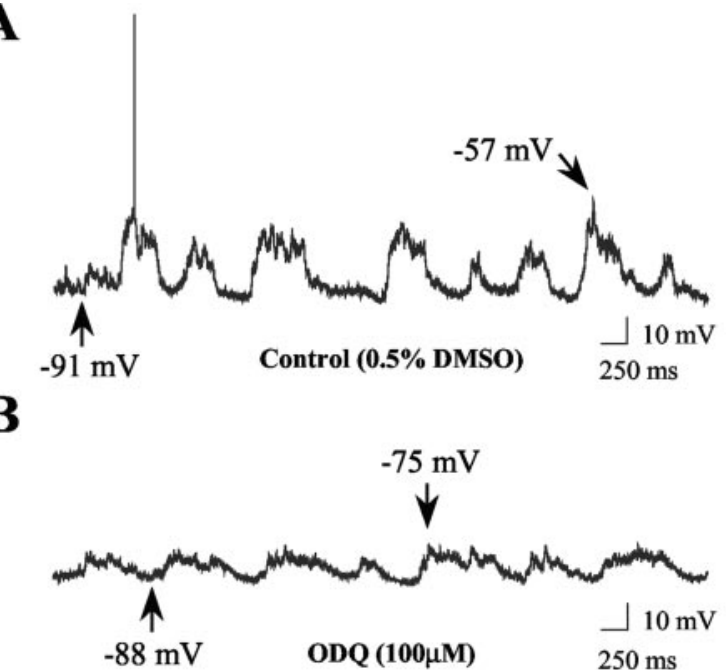

C

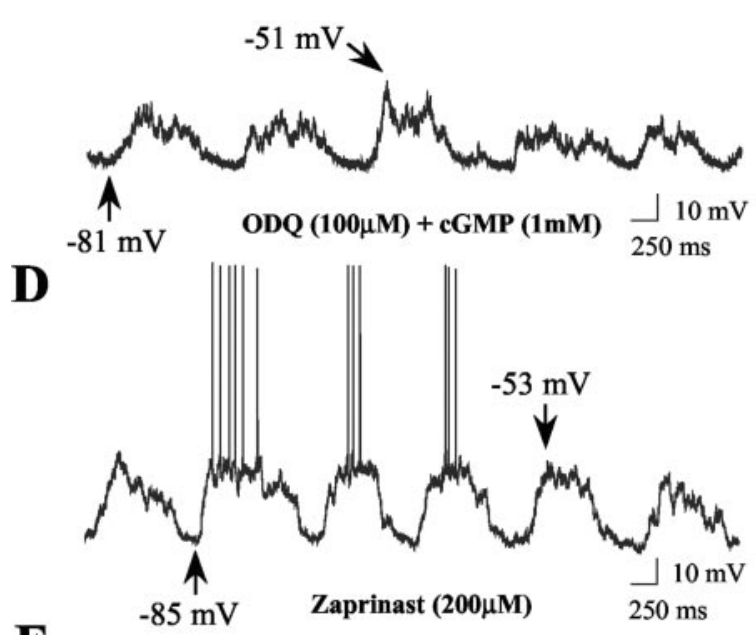

$\mathbf{E}$

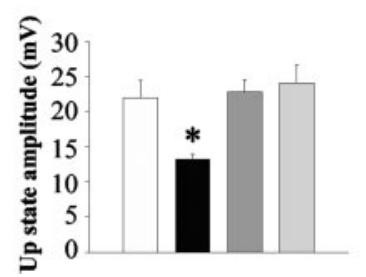

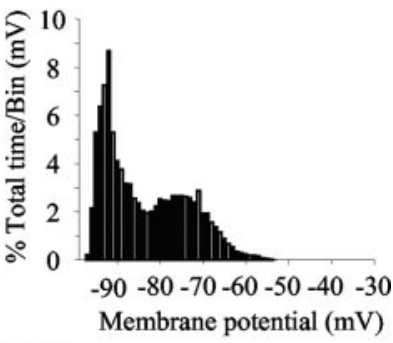
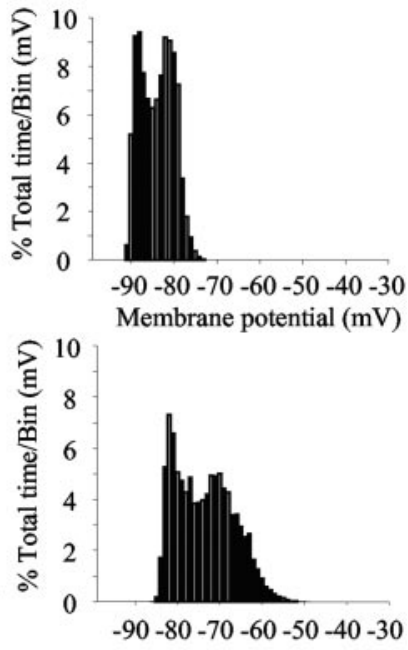

Membrane potential $(\mathrm{mV})$

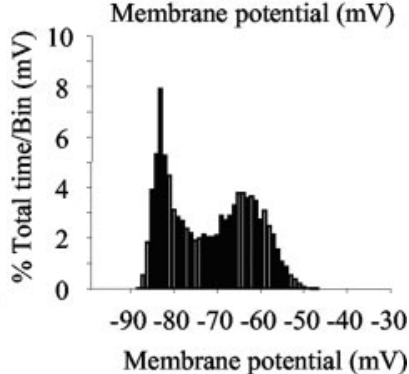

Figure 7. Manipulation of intracellular cGMP levels modulates the membrane activity of striatal neurons. Striatal neurons were recorded after intracellular application ( $\sim 5 \mathrm{~min})$ of either vehicle (0.5\% DMSO), the GC inhibitor ODQ (100 $\mu \mathrm{M}), 0 \mathrm{DQ}$ plus CGMP (1 mm), or the PDE inhibitor zaprinast $(200 \mu \mathrm{M})$. A, Left, After vehicle injection, striatal neurons $(n=6)$ exhibited typical rapid spontaneous shifts in steady-state membrane potential and irregular spontaneous spike discharge. Right, Time interval plots of membrane potential activity recorded from control neurons demonstrated bimodal membrane potential distributions indicative of bistable membrane activity. $B$, Left, Striatal neurons recorded after ODQ injection $(n=5)$ exhibited significantly lower amplitude up events compared with vehicle-injected controls and rarely fired action potentials. Right, The depolarized portion of the membrane potential distribution of neurons recorded after ODQ injection was typically shifted leftward (hyperpolarized) compared with controls. C, Left, Striatal neurons recorded after ODQ and CGMP injection rarely fired action potentials but exhibited high amplitude up events with extraordinarily long durations. Right, The membrane potential distribution of neurons recorded after ODQ and CGMP injection was similar to controls, indicating that CGMP partially reversed some of the effects of ODQ. D, Left, Striatal neurons recorded after intracellular injection of zaprinast $(n=5)$ exhibited high amplitude up events with extraordinarily long durations. Additionally, all of the cells fired action potentials at relatively high rates $(0.4-2.2 \mathrm{~Hz})$. Right, The membrane potential distribution of these neurons was typically shifted rightward (depolarized) compared with controls. Arrows indicate the membrane potential at its maximal depolarized and hyperpolarized levels. $E$, Left, The mean \pm SEM up-state amplitude was reduced after intracellular injection of $\mathrm{ODQ}\left(n=5\right.$; ${ }^{*} p<0.05$; ANOVA with Dunnett's post hoc test). Right, The mean \pm SEM up-state duration was significantly prolonged during $\mathrm{CGMP} / 0 \mathrm{DQ}$ and zaprinast injections $\left(n=5 ;{ }^{*} p<0.05\right.$; ANOVA with Dunnett's post hoc test). 
Table 2. Effects of intracellular manipulations of cGMP levels on the membrane properties of striatal neurons

\begin{tabular}{|c|c|c|c|c|}
\hline Membrane properties & Control & ODQ & $\mathrm{ODQ}+\mathrm{CGMP}$ & Zaprinast \\
\hline Firing rate $(\mathrm{Hz})$ & $0.54 \pm 0.33$ & $0.06 \pm 0.06$ & $0.05 \pm 0.04$ & $\begin{array}{c}0.91 \pm 0.35^{* *} \\
(p<0.05)\end{array}$ \\
\hline Average MP (mV) & $-78.2 \pm 2.7$ & $-84.7 \pm 2.3$ & $-79.2 \pm 2.7$ & $\begin{array}{c}-65.0 \pm 0.76^{*} \\
(p<0.05)\end{array}$ \\
\hline Up-state frequency $(\mathrm{Hz})$ & $1.17 \pm 0.11$ & $1.03 \pm 0.14$ & $0.90 \pm 0.06$ & $0.97 \pm 0.06$ \\
\hline Maximal depolarized $\mathrm{MP}^{a}(\mathrm{mV})$ & $-55.0 \pm 3.5$ & $\begin{array}{c}-72.2 \pm 2.5^{*} \\
\quad(p<0.05)\end{array}$ & $-55.0 \pm 2.0$ & $\begin{array}{c}-44.4 \pm 2.7^{*} \\
\quad(p<0.05)\end{array}$ \\
\hline Up-state mode (mV) & $-73.0 \pm 4.2$ & $-81.4 \pm 4.0$ & $-75.8 \pm 1.8$ & $\begin{array}{c}-61.2 \pm 1.7^{*} \\
\quad(p<0.05)\end{array}$ \\
\hline Down-state mode (mV) & $-83.0 \pm 3.8$ & $-87.4 \pm 4.6$ & $-87.0 \pm 1.4$ & $-72.0 \pm 3.7$ \\
\hline Maximal hyperpolarized MP (mV) & $-91.3 \pm 3.2$ & $-90.8 \pm 3.1$ & $-91.8 \pm 1.4$ & $\begin{array}{c}-80.4 \pm 2.9^{*} \\
\quad(p<0.05)\end{array}$ \\
\hline Input resistance $(\mathrm{m} \Omega$ ) & $34.3 \pm 1.8$ & $\begin{array}{l}17.7 \pm 3.5^{*} \\
(p<0.05)\end{array}$ & $20.3 \pm 1.0$ & $28.4 \pm 1.8$ \\
\hline \multicolumn{5}{|l|}{ Action potential } \\
\hline Amplitude (mV) & $70.0 \pm 2.4$ & $\begin{array}{l}60.0 \pm 2.4^{*} \\
(p<0.05)\end{array}$ & $68.2 \pm 3.1$ & $71.6 \pm 2.5$ \\
\hline Overshoot (mV) & $+22.1 \pm 2.8$ & $+14.7 \pm 2.7$ & $+22.5 \pm 1.6$ & $+25.9 \pm 3.5$ \\
\hline Duration (msec) & $1.2 \pm 0.08$ & $1.3 \pm 0.05$ & $1.1 \pm 0.07$ & $1.2 \pm 0.05$ \\
\hline Threshold (mV) & $-47.5 \pm 1.2$ & $-45.7 \pm 3.0$ & $-45.6 \pm 2.2$ & $-45.7 \pm 1.5$ \\
\hline $\mathrm{AHP}(\mathrm{mV})$ & $-12.5 \pm 1.6$ & $-12.3 \pm 2.4$ & $-12.9 \pm 0.4$ & $-10.9 \pm 1.4$ \\
\hline
\end{tabular}

All data are means \pm SEM from $n=5-6$ neurons. MP, Membrane potential; AHP, afterhyperpolarization.

*Statistical significance was determined by comparing DMSO control to drug groups using an ANOVA with Dunnett's post hoc test.

**Significantly different compared to the ODQ group using an ANOVA with Dunnett's post hoc test.

${ }^{a}$ This calculation does not include membrane potential fluctuations contributed by action potentials.

responsiveness of striatal MSNs to both hyperpolarizing current pulses and excitatory inputs involved in facilitating up events. Furthermore, intracellular injection of a GC inhibitor decreased the responsiveness of MSNs to depolarizing current in a manner that was reversed by coinjection of cGMP. Conversely, augmentation of endogenous intracellular cGMP levels induced via injection of a PDE inhibitor robustly increased membrane excitability. Together, these findings indicate that NO-producing interneurons play an important role in enhancing the membrane excitability of striatal MSNs in vivo via the activation of GC signaling cascades.

\section{Regulation of striatal spiny neuron activity by tonic NO signaling}

A particularly interesting finding was that both extracellular infusion of the NO scavenger and intracellular injection of the GC inhibitor induced large decreases in the input resistance of MSNs, which were partially reversed via coadministration of cGMP analogs. Currently, little is known regarding the influence of cGMP and PKG on specific ion channels of striatal MSNs. However, previous studies have shown that cGMP analogs depress the activity of brain inwardly rectifying $\mathrm{K}^{+}$channels expressed in $\mathrm{Xe}$ nopus oocytes (Ito et al., 1997) and inhibit $\mathrm{GABA}_{\mathrm{A}}$ currents in cultured amacrine cells (Wexler et al., 1998). Given that the ODQ-induced decrease in input resistance observed in the current study coincided with a decrease in membrane excitability and a hyperpolarization of the membrane potential, it is possible that tonic cGMP levels normally suppress the activity of $\mathrm{K}^{+}$ channels in MSNs. Removal of this influence after ODQ injection would increase the membrane permeability of $\mathrm{K}^{+}$and decrease membrane excitability. Although speculative, this increase in membrane permeability could also induce a shunting of $\mathrm{Na}^{+}$ ions and lead to the reversible decrease in the amplitude of naturally occurring up states and evoked action potentials observed after ODQ administration.

A role for NO-GC signaling in modulating the membrane activity of MSNs is supported by ultrastructural studies demon- strating that NOS-immunoreactive processes form synaptic contacts primarily on dendritic spine shafts (Calabresi et al., 1999b; Sancesario et al., 2000; Hidaka and Totterdell, 2001). MSNs have also been shown to contain high levels of GC, cGMP, PKG (Ariano, 1983), and dopamine- and cAMP-regulated phosphoprotein-32 (DARPP-32) (Greengard et al., 1999). Additionally, studies using organotypic cultures have demonstrated that DARPP-32-containing striatal MSNs are innervated by a dense plexus of NOS-positive processes (Gomez-Urquijo et al., 1999). Studies in vitro have also shown that the NO-dependent stimulation of cGMP-dependent protein kinase activity results in increased DARPP-32 phosphorylation in striatonigral nerve terminals (Tsou et al., 1993). Recent electrophysiological studies performed in brain slices have demonstrated that this NO-GCmediated increase in DARPP-32 phosphorylation is critically involved in the induction of long-term synaptic depression in corticostriatal pathways (Calabresi et al., 1999a,b, 2000b).

\section{NO-GC modulation of up- and down-state membrane potential fluctuations and GLUergic neurotransmission}

The current in vivo intracellular studies indicate that NO-GC signaling may play a major role in controlling the excitability of striatal neurons from both the up and down membrane potential states. Although the triggering of the up state is known to be dependent on GLUergic inputs (Wilson, 1993; O'Donnell and Grace, 1995; Wilson and Kawaguchi, 1996), little is known regarding the role of neuromodulators in regulating the amplitude and duration of up events. Given the dual role for GLUergic neurotransmission in generating the depolarized up state in MSNs, and stimulating both spike activity (Kawaguchi, 1993) and NO production in NOS interneurons (Marin et al., 1992; East et al., 1996), we reasoned that endogenous NO may play a role in regulating the up- and down-state membrane potential characteristics of MSNs. In support of this, antagonists of the NO-cGMP signaling pathway were observed to attenuate the amplitude of spontaneous up events and synaptic responses 
$\mathbf{A}$

\section{Control (0.5\% DMSO)}
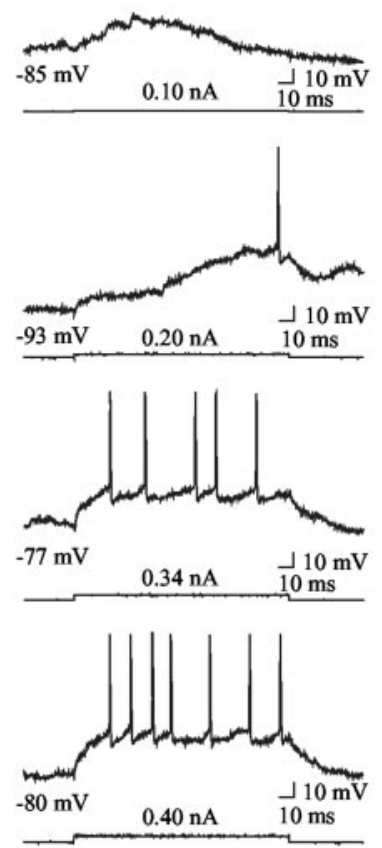

\section{ODQ $(0.5 \%$ DMSO)}
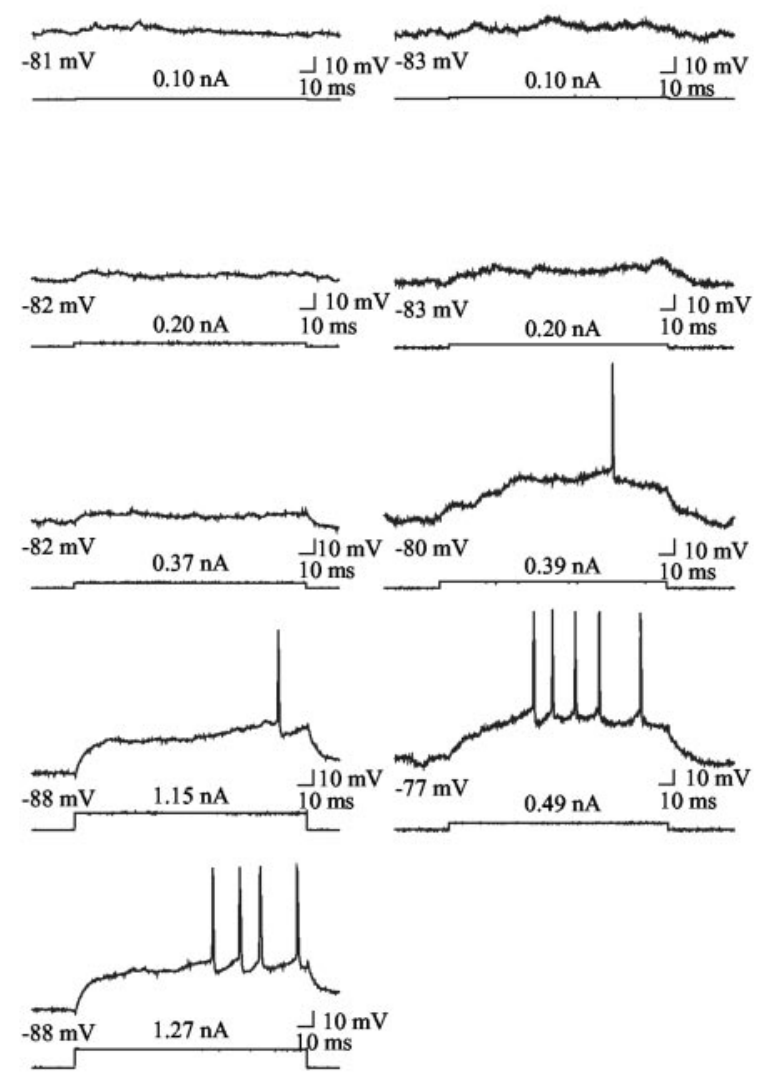

B
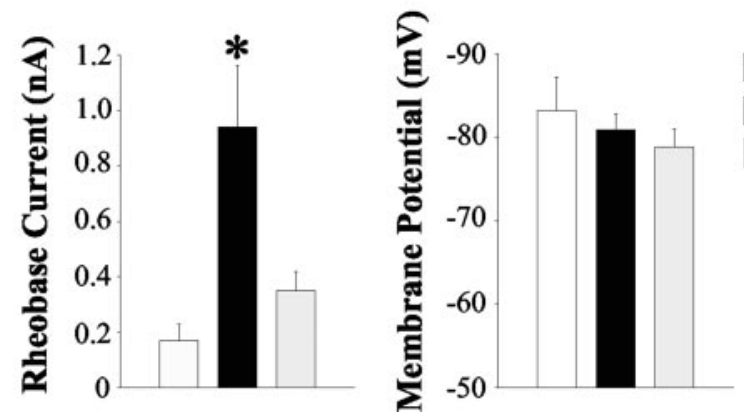

Figure 8. Intracellular injection of the guanylyl cyclase inhibitor ODQ decreased the responsiveness of striatal neurons to intracellular current injection. $A$, Left column, Response of a single cell to increasing amplitudes of intracellular current injected after vehicle (0.5\% DMSO) injection. Middle column, Decreased responsiveness of a typical single cell to depolarizing current pulses injected after ODQ injection ( $\sim 5-6 \mathrm{~min})$. Note that the current amplitude required to reach threshold (rheobase) is significantly larger $(1.15 \mathrm{nA})$ than the representative control $(0.20 \mathrm{nA})$. Right column, Partial reversal of the ODQ effect after coinjection with CGMP ( 5-6 min). Bottom traces indicate current injection steps. Top traces indicate the voltage response. The membrane potential before current injection is indicated below the voltage trace. The current amplitude is indicated above the current step. $B$, Left, The mean \pm SEM current amplitude required to reach threshold (rheobase) was dramatically increased in cells recorded after intracellular injection of ODQ $\left({ }^{*} p<0.05\right.$; ANOVA with Dunnett's post hoc test) compared with controls $(0.5 \%$ DMSO) and neurons recorded after ODQ and CGMP injection. Right, The mean \pm SEM membrane potential of the same striatal neurons recorded before current injection was not significantly different across treatment groups ( $p>0.05)$.

MSNs measured in both the up and down states and prolonged the duration of the up state.

The observations that NO antagonist administration reduced the amplitude of naturally occurring up events and evoked synaptic responses indicate that the activation of NO signaling cascades may increase the activity of spiny projection cells via amplification of glutamatergic transmission. Given that intracellular injection of the GC inhibitor also potently decreased the amplitude of up events and input resistance, one might argue that the NO-mediated amplification of GLUergic neurotransmission is occurring postsynaptically. However, microdialysis studies have demonstrated that low levels of cGMP are normally present in the striatal extracellular space (Globus et al., 1995). Striatal extracellular cGMP levels are also significantly decreased after NOS inhibition, indicating that they are maintained by tonic NO signaling (Globus et al., 1995). Together with studies showing that reverse dialysis of NO generators (West et al., 2002a) and cGMP analogs (Guevara-Guzman et al., 1994) increases extracellular glutamate levels, these observations suggest that cGMP may also act as an intercellular signaling molecule involved in regulating presynaptic GLUergic neurotransmission. Our findings showing that intrastriatal NO scavenger infusion attenuated synaptic facilitation induced by paired-pulse stimulation of corticostriatal pathways also suggest that a component of the NO-mediated enhancement of glutamatergic neurotransmission may be occurring via a presynaptic mechanism.

Functional implications: role of NO effector pathways in normal motor behavior and parkinsonian states Although the interaction between NOS interneurons and MSNs and their respective corticostriatal and nigrostriatal afferents remains to be fully characterized, these circuits appear to be critically involved in the generation of motor behavior. Thus, inhibition of NOS in rodents decreases basal motor activity (Stewart et al., 1994) and movement stimulated by substance $\mathrm{P}$ (Mancuso et al., 1994), MK-801 (Deutsch et al., 1996), and DA $D_{1}$ and $D_{2}$ agonists (Starr and Starr, 1995). Systemic administration of NOS inhibitors also potentiates catalepsy induced by $\mathrm{D}_{2}$ antagonists (Ca-

evoked by electrical stimulation of the oPFC. Additionally, intracellular application of a GC inhibitor was observed to decrease the responsiveness of MSNs to depolarizing current pulses delivered in the down state. Conversely, pharmacological inhibition of PDEs involved in metabolizing cGMP induced a profound depolarizing influence on several of the membrane characteristics of vas and Navarro, 2002). Consistent with our observations with zaprinast, a recent study has shown that enhancement of cyclic nucleotide signaling in PDE $1 \mathrm{~B}$ knock-out mice results in locomotor hyperactivity and augmented DARPP-32 phosphorylation in response to DA agonists (Reed et al., 2002). These findings suggest a role for both DA-stimulated cAMP and NO-stimulated 
cGMP in the generation of movement. Moreover, recent studies demonstrate that the activity of striatal NOS is depressed in 6-OHDA-lesioned animals (de Vente et al., 2000; Sahach et al., 2000), and NOS cell numbers and mRNA are significantly reduced in postmortem parkinsonian brains (Bockelmann et al., 1994; Eve et al., 1998). The above reports, together with studies showing that NO facilitates both tonic and phasic DA neurotransmission in intact animals (West et al., 2002a) and stimulates L-3,4-dihydroxyphenylalanine release from striatal tissues (Sanchez et al., 2002), suggest that agents or treatments designed to augment striatal NOS activity may be useful as antiparkinsonian therapies.

\section{References}

Ariano MA (1983) Distribution of the components of the guanosine $3^{\prime}, 5^{\prime}$ phosphate system in rat caudate-putamen. Neuroscience 10:707-723.

Bockelmann R, Wolf G, Ransmayr G, Riederer P (1994) NADPH-diaphorase/nitric oxide synthase containing in normal and Parkinson's disease putamen. J Neural Transm Park Dis Dement Sect 7:115-121.

Calabresi P, Gubellini P, Centonze D, Sancesario G, Morello M, Giorgi M, Pisani A, Bernardi G (1999a) A critical role of the nitric oxide/cGMP pathway in corticostriatal long-term depression. J Neurosci 19:2489-2499.

Calabresi P, Centonze D, Gubellini P, Marfia GA, Bernardi G (1999b) Glutamate-triggered events inducing corticostriatal long-term depression. J Neurosci 19:6102-6110.

Calabresi P, Centonze D, Gubellini P, Marfia GA, Pisani A, Sancesario G, Bernardi G (2000a) Synaptic transmission in the striatum: from plasticity to neurodegeneration. Prog Neurobiol 61:231-265.

Calabresi P, Gubellini P, Centonze D, Picconi B, Bernardi G, Chergui K, Svenningsson P, Fienberg A, Greengard P (2000b) Dopamine and cAMP-regulated phosphoprotein $32 \mathrm{kDa}$ controls both striatal long-term depression and long-term potentiation, opposing forms of synaptic plasticity. J Neurosci 20:8443-8451.

Cavas M, Navarro JF (2002) Coadministration of L-NOARG and tiapride: effects on catalepsy in male mice. Prog Neuropsychopharmacol Biol Psychiatry 26:69-73.

Charpier S, Deniau JM (1997) In vivo activity-dependent plasticity at cortico-striatal connections: evidence for physiological long-term potentiation. Proc Natl Acad Sci USA 94:7036-7040.

Deniau JM, Menetrey A, Charpier S (1996) The lamellar organization of the rat substantia nigra pars reticulata: segregated patterns of striatal afferents and relationship to the topography of corticostriatal projections. Neuroscience 73:761-781.

Deutsch SI, Rosse RB, Paul SM, Tomasino V, Koetzner L, Morn CB, Mastropaolo J (1996) 7-Nitroindazole and methylene blue, inhibitors of neuronal nitric oxide synthase and NO-stimulated guanylyl cyclase, block MK-801 elicited behaviors in mice. Neuropsychopharmacology 15:37-43.

de Vente J, Markerink-van Ittersum M, Van Abeelen J, Emson PC, Axer H, Steinbusch HWM (2000) NO-mediated cGMP synthesis in cholinergic neurons in the rat forebrain: effects of lesioning dopaminergic or serotonergic pathways on nNOS and cGMP synthesis. Eur J Neurosci 12:507-519.

Di Giovanni G, Ferraro G, Sardo P, Galati S, Esposito E, La Grutta V (2003) Nitric oxide modulates striatal neuronal activity via soluble guanylyl cyclase: an in vivo microiontophoretic study in rats. Synapse 48:100-107.

East JS, Parry-Jones A, Brotchie JM (1996) Ionotropic glutamate receptors and nitric oxide in the rat striatum. NeuroReport 8:71-75.

Eve DJ, Nisbet AP, Kingsbury AE, Hewson EL, Daniel SE, Lees AJ, Marsden CD, Foster OJ (1998) Basal ganglia neuronal nitric oxide synthase mRNA expression in Parkinson's disease. Mol Brain Res 63:62-71.

Garthwaite J, Boulton CL (1995) (1995) Nitric oxide signaling in the central nervous system. Annu Rev Physiol 57:683-706.

Globus MY, Prado R, Busto R (1995) Ischemia-induced changes in extracellular levels of striatal cyclic GMP: role of nitric oxide. NeuroReport 6:1909-1912.

Gomez-Urquijo SM, Hokfelt T, Ubink R, Lubec G, Herrera-Marschitz M (1999) Neurocircuitries of the basal ganglia studied in organotypic cul- tures: focus on tyrosine hydroxylase, nitric oxide synthase, and neuropeptide immunocytochemistry. Neuroscience 94:1133-1151.

Greengard P, Allen PB, Nairn AC (1999) Beyond the dopamine receptor: the DARPP-32/protein phosphatase-1 cascade. Neuron 23:435-447.

Guevara-Guzman R, Emson PC, Kendrick KM (1994) Modulation of in vivo striatal transmitter release by nitric oxide and cyclic-GMP. J Neurochem 62:807-810.

Hidaka S, Totterdell S (2001) Ultrastructural features of the nitric oxide synthase-containing interneurons in the nucleus accumbens and their relationship with tyrosine hydroxylase containing terminals. J Comp Neurol 431:139-154.

Ito H, Tsuchimochi H, Tada Y, Kurachi Y (1997) Phosphorylationindependent inhibition by intracellular cyclic nucleotides of brain inwardly rectifying $\mathrm{K}^{+}$current expressed in Xenopus oocytes. FEBS Lett 402:12-16.

Kawaguchi Y (1993) Physiological, morphological, and histochemical characterization of three classes of interneurons in rat neostriatum. J Neurosci 13:4908-4923.

Kawaguchi Y (1997) Neostriatal cell subtypes and their functional roles. Neurosci Res 27:1-8.

Kharazia VN, Schmidt HHW, Weinberg RJ (1994) Type 1 nitric oxide synthase fully accounts for NADPH-diaphorase in rat striatum but not cortex. Neuroscience 62:983-987.

Kubota Y, Mikawa S, Kawaguchi Y (1993) Neostriatal GABAergic interneurons contain NOS, calretinin or parvalbumin. NeuroReport 5:205-208.

Mancuso F, Calignano A, Sorrentino L (1994) Endogenous nitric oxide modulates the behavioral effects elicited by substance P in rat. Eur J Pharmacol 271:329-333.

Marin P, Lafon-Cazal M, Bockaert J (1992) A nitric oxide activity selectively stimulated by NMDA receptors depends on protein kinase $\mathrm{C}$ activation in mouse striatal neurons. Eur J Neurosci 4:425-432.

O’Donnell P, Grace AA (1995) Synaptic interactions among excitatory afferents to nucleus accumbens neurons: hippocampal gating of prefrontal cortical input. J Neurosci 15:3622-3639.

O'Donnell P, Grace AA (1997) Cortical afferents modulate striatal gap junction permeability via nitric oxide. Neuroscience 76:1-5.

Paxinos G, Watson C (1986) The rat brain in stereotaxic coordinates. New York: Academic.

Reed TM, Repaske DR, Snyder GL, Greengard P, Vorhees CV (2002) Phosphodiesterase $1 \mathrm{~B}$ knock-out mice exhibit exaggerated locomotor hyperactivity and DARPP-32 phosphorylation in response to dopamine agonists and display impaired spatial learning. J Neurosci 22:5188-5197.

Sahach VF, Baziliuk OV, Oleshko MM, Kotsiuruba OV, Bukhanevych OM, Appenzeller O (2000) The nitric oxide system in a chronic deficiency of mesostriatal dopamine: the action of nitroglycerin. Fiziol Zh 46:55-63.

Sancesario G, Morello M, Reiner A, Giacomini P, Massa R, Schoen S, Bernardi G (2000) Nitrergic neurons make synapses on dual-input dendritic spines of neurons in the cerebral cortex and the striatum of the rat: implications for a postsynaptic action of nitric oxide. Neuroscience 99:627-642.

Sanchez JJ, Abreu P, Gonzalez C (2002) Sodium nitroprusside stimulates L-DOPA release from striatal tissue through nitric oxide and cGMP. Eur J Pharmacol 438:79-83.

Starr MS, Starr BS (1995) Do NMDA receptor-mediated changes in motor behavior involve nitric oxide? Eur J Pharmacol 272:211-217.

Stewart J, Deschamps S-E, Amir S (1994) Inhibition of nitric oxide synthase does not block the development of sensitization to the behavioral activating effects of amphetamine. Brain Res 641:141-144.

Tsou K, Snyder G, Greengard P (1993) Nitric oxide/cGMP pathway stimulates phosphorylation of DARPP-32, a dopamine and cAMP-regulated phosphoprotein in the substantia nigra. Proc Natl Acad Sci USA 90:3462-3465.

West AR, Galloway MP (1997) Endogenous nitric oxide facilitates striatal dopamine and glutamate efflux in vivo: role of ionotropic glutamate receptor-dependent mechanisms. Neuropharmacology 36:1571-1581.

West AR, Grace AA (2000) Striatal nitric oxide signaling regulates the neuronal activity of midbrain dopamine neurons in vivo. J Neurophysiol 83:1796-1808.

West AR, Grace AA (2001) Endogenous nitric oxide modulates the excitability of striatal neurons exhibiting bistable membrane potentials: studies using combined in vivo intracellular recordings and microdialysis in vivo. Soc Neurosci Abstr 27:31.5. 
West AR, Grace AA (2002a) Opposite influences of endogenous dopamine $D_{1}$ and $D_{2}$ receptor activation on activity states and electrophysiological properties of striatal neurons: studies combining in vivo intracellular recordings and reverse microdialysis. J Neurosci 22:294-304.

West AR, Grace AA (2002b) Guanylyl cyclase modulates the excitability of striatal neurons exhibiting bistable membrane potentials in vivo. Soc Neurosci Abstr 28:249.17.

West AR, Galloway MP, Grace AA (2002a) Regulation of striatal dopamine neurotransmission by nitric oxide: effector pathways and signaling mechanisms. Synapse 44:227-245.

West AR, Moore HM, Grace AA (2002b) Direct examination of local regulation of membrane activity in striatal and prefrontal cortical neurons in vivo using simultaneous intracellular recording and microdialysis. J Pharmacol Exp Ther 301:867-877.

Wexler EM, Stanton PK, Nawy S (1998) Nitric oxide depresses GABA $A_{A}$ receptor function via coactivation of cGMP-dependent kinase and phosphodiesterase. J Neurosci 18:2342-2349.

Wilson CJ (1993) The generation of natural firing patterns in neostriatal neurons. In: Chemical signaling in the basal ganglia: progress in brain research (Arbuthnott GW and Emson PC, eds), pp 277-297. Amsterdam: Elsevier.

Wilson CJ, Kawaguchi Y (1996) The origins of two-state spontaneous membrane potential fluctuations of neostriatal spiny neurons. J Neurosci 16 : $2397-2410$ 